\title{
Enhanced Characteristic Vibration Signal Detection of Generator Based on Time-Wavelet Energy Spectrum and Multipoint Optimal Minimum Entropy Deconvolution Adjusted Method
}

\author{
Yu-Ling He $\left(\mathbb{D}\right.$, Tao Wang, Kai Sun, Xiao-Long Wang $\mathbb{D}^{D}$, Bo Peng, and Shu-Ting Wan \\ Department of Mechanical Engineering, North China Electric Power University, Baoding, Hebei 071003, China \\ Correspondence should be addressed to Xiao-Long Wang; wangxiaolong0312@126.com
}

Received 20 November 2019; Revised 15 March 2020; Accepted 19 March 2020; Published 20 April 2020

Academic Editor: Carlo Renno

Copyright (c) $2020 \mathrm{Yu}$-Ling He et al. This is an open access article distributed under the Creative Commons Attribution License, which permits unrestricted use, distribution, and reproduction in any medium, provided the original work is properly cited.

\begin{abstract}
To overcome the shortage of low SNR (signal to noise ratio) of the multipole generator vibration signal which brings rigid difficulty to the fault diagnosis, a new method which combines the Time-Wavelet Energy Spectrum (TWES) with the Multipoint Optimal Minimum Entropy Deconvolution Adjusted (MOMEDA) algorithm is proposed. This method uses TWES to extract and enhance the characteristic signal, while employing MOMEDA to optimize the spectrum structure and filter the noise. The application of this method to the simulating signal as well as the test stator vibration signal in a 6-pole generator before and after rotor interturn short circuit fault validates the effectiveness of the method. Moreover, the comparison among the proposed method and some other general methods such as the Empirical Mode Decomposition (EMD) and the maximum correlative kurtosis deconvolution (MCKD) suggests that the proposed method is superior to these methods.
\end{abstract}

\section{Introduction}

The generator is a complex system running in a strict condition. Scholars have taken a lot of energy in exploring different means for high-accuracy monitoring and fast fault diagnosis. Among these means, the vibration based method has attracted much preference due to its easy signal acquisition and rich fault information [1],2]. This method has been proposed and developed for a long time even since the generator was invented.

Recently, signal processing field has gained a rapid development and achieved many new outputs. For instance, to overcome the shortcomings of Fourier Transform/Fast Fourier Transform, people proposed the Wavelet Transform (WT) method which has the advantages in both the localization and adaptive window width [2-5]. However, this method needs to choose the proper mother wavelet and set the decomposition layers. Although scholars have tried to improve this method by different means, for example, the Adaptive Reinforced Empirical Morlet Wavelet Transform [6], this method still has drawback. Alternatively, people proposed the Empirical Mode Decomposition (EMD) method to adaptively decompose the signal [7-11]. But this method came across the mode mixture and the end-effect problems. Subsequently, scholars further proposed the ensemble empirical mode decomposition (EEMD) method [12-14] to make the signal distributed in a proper reference scale and the decomposition more accurate.

Actually, the signal delivering process can be treated as the linear convolution of the original signal and the transfer channel [15], while the extraction of the faulty signal can be treated as the deconvolution procedure. In this view, scholars proposed the Minimum Entropy Deconvolution (MED) method [16-18] to enhance the impulse component of the signal. However, it is found that this method has some inevitable shortcomings. For instance, it is not a globally optimal filter but only a locally optimal one which can only obtain part of the impulse signal. To overcome the side-effect of MED, Mcdonald and Zhao [19] proposed a special method named Multipoint Optimal Minimum Entropy Deconvolution Adjusted (MOMEDA) which employs the objective vector together with several $D$ norms, and had obtained wide application [19-24].

However, most of these aforementioned methods are proposed for the fault diagnosis of rolling bearings, while few 
of them are specifically used for the generators. Unfortunately, the application of these methods to generators, especially to the multipole generators does not gain ideally satisfied effect since much weaker impulse features but meanwhile too many components (including both frictional components and multiple harmonics of the basic electrical frequency) are included in the generator's vibration signal due to the large mass of the generator set and the abundant influential factors such as the thermal and the electromagnetic forces (affected by not only mechanical factors but also electrical and other excitations). In other words, the vibration signal of the generator has different properties from that of rolling bearings. The energy in the vibration signal is more dispersive and the ratio of signal to nose (RSN) is much smaller. It is therefore more critical for the generator vibration signals to enhance the impulse features by effective algorithms.

Actually, the MOMEDA method is a good tool to enhance the impulse features of the vibration signal, but the authors found it not enough for the multipole generators. It is found in $[25,26]$ that the radial stator vibration components at $f, 2 f, 3 f$, and $4 f$ ( $f$ is the fundamental frequency) are the Characteristic frequency components under the rotor interturn short circuit faults. However, in practical performing circumstance, these four components are inferior to other components in the spectrum, especially when the fault degree is not so serious. In order to further enhance the impulse features of the generator's vibration signal, in this paper, we proposed the Timewavelet Energy Spectrum (TWES) method and combine it with the Multipoint Optimal Minimum Entropy Deconvolution Adjusted (MOMEDA) method for an improvement. This proposed method is applied to process the simulating signal as well as the test stator vibration signal of a 6-pole synchronous generator to diagnose the rotor interturn short circuit faults as a validation.

The remainder of this paper is constructed as follows. The algorithm of the proposed method is explained in Section 2, while the comprehensive processing results and the processing comparisons between the proposed method and other general methods for the simulating signal and the test signal are illustrated in Section 3 and Section 4, respectively. Finally, primary conclusions obtained from the study are drawn up in Section 5.

\section{Algorithm of Proposed Method}

2.1. Time-Wavelet Energy Spectrum. For an energy limited signal, its wavelet transform can be written as

$$
W T_{x}(a, b)=\left\langle x(t), \psi_{a, b}(t)\right\rangle=\frac{1}{\sqrt{a}} \int_{R} x(t) \psi^{*}\left(\frac{t-b}{a}\right) \mathrm{d} t,
$$

where $\langle x, y\rangle$ is the convolution operation to $x$ and $y, \psi^{*}(t)$ is the conjugate of the mother wavelet $\psi(t)$, and $\psi_{a, b}(t)$ is the wavelet basis function produced by the scale performance and translation transformation to $\psi(t)$, which can be written as

$$
\psi_{a, b}(t)=|a|^{-(1 / 2)} \psi\left(\frac{t-b}{a}\right),
$$

where $a(a \neq 0)$ is the scale factor related to the frequency and the scale performance, while $b$ is the location parameter related to the time and the translation transformation.

For a better comparison, here we also list the definition of Fourier Transform which can be written as

$$
F(\omega)=\int_{R} f(t) e^{-i \omega t} \mathrm{~d} t
$$

where $f(t)$ is the time-domain signal, and $\omega$ is the angular frequency.

Obviously, there is only one independent variable $\omega$ and therefore can display only the frequency information. However, there are two variables $a$ and $b$ which can display both the frequency and the time information at the same time for the Wavelet Transform. The significance of these two variables is that it offers the exact time for a specific frequency.

According to the energy conversion principles, it has

$$
\int_{-\infty}^{+\infty}|x(t)|^{2} \mathrm{~d} t=\frac{1}{C_{\psi}} \int_{-\infty}^{+\infty} \int_{-\infty}^{+\infty}\left|W T_{x}(a, b)\right|^{2} \frac{\mathrm{d} a \mathrm{~d} b}{a^{2}}
$$

where $\left|W T_{x}(a, b)\right|^{2}$ is the scale figure which is actually the energy distribution depending on the scale factor $a$ and the shift factor $b$. It can be further modified to

$$
\left\{\begin{array}{l}
\int_{-\infty}^{+\infty}|x(t)|^{2} \mathrm{~d} t=\frac{1}{C_{\psi}} \int_{-\infty}^{+\infty} E_{b} \mathrm{~d} b, \\
E_{b}=\int_{-\infty}^{+\infty} \frac{\left|W T_{x}(a, b)\right|^{2}}{a^{2}} \mathrm{~d} a,
\end{array}\right.
$$

where $E_{b}$ is defined as the Time-wavelet Energy Spectrum (TWES) which reflects the energy distribution of the signal versus time. In a particular moment when there is an impulse, there will be a peak appear in TWES. In this view, the impulse characteristics can be extracted via spectrum analysis on the energy distribution.

Since the wavelet transform effect is defectively dependent on the wavelet basis function, it is significant to select a proper wavelet basis function which has the similar impulse properties as the original signal. It is found in [27] that the Morlet function is one of the optimal selections. This function is of square exponential decay and can be expressed as

$$
\psi(t)=\pi^{-(1 / 4)}\left[\exp \left(-i \omega_{0} t\right)-\exp \left(-\frac{\omega_{0}^{2}}{2}\right)\right] \exp \left(-\frac{t^{2}}{2}\right),
$$

where $\omega_{0}$ is the central frequency.

\subsection{Processing Method Based on TWES and MOMEDA.} The actual vibration signal can be expressed as

$$
x=r * y+u,
$$

where $x$ is the acquisition signal, $y$ is the impulse signal which includes the faulty information, $r$ is the transfer function of the whole system, and $u$ is the disturbing signal.

The function of MOMEDA is to reconstruct the impulse signal $y$ by a noninteractive means with the optimal filter $f$, so that the impact of the noise on the original signal extraction 
can be maximally depressed. The deconvolution can be expressed as

$$
y=f * x=\sum_{k=1}^{N-L} f_{k} x_{k+L-1},
$$

where $k=1,2, \ldots, N-L, N$ is the data length of $f, L$ is the original shift distance between $f$ and $x$.

To obtain a better effect, the maximization problem is introduced into MOMEDA by proposing multipoint $D$ norms which can be written as

$$
\left\{\begin{array}{l}
\text { Multi } D-\text { Norm }=\operatorname{MDN}(y, t)=\frac{t^{T} y}{\|y\|}, \\
\text { MOMEDA }=\max _{f} \operatorname{MDN}(y, t)=\max _{f} \frac{t \cdot y}{\|y\|},
\end{array}\right.
$$

where $t$ is a constant vector that defines both the location and the weight factor of the objective impulse for deconvolution, $y$ is the signal, and $T$ is the deconvolution period (also the faulty signal period). The more similar $t$ is to $y$, the better the deconvolution effect will be. The best solution will appear when the multipoint $D$ norms obtain the max value. At this moment, the group of filters is the optimal one. Then, the solution to equation (9) converts to

$$
\frac{\mathrm{d}}{\mathrm{d} f}\left(\frac{t^{T} y}{\|y\|}\right)=0,
$$

where $f=f_{1}, f_{2}, \ldots, f_{L} ; t=t_{1}, t_{2}, \ldots, t_{N-L}$

Based on (9) and (10) there is

$$
\frac{\mathrm{d}}{\mathrm{d} f}\left(\frac{t^{T} y}{\|y\|}\right)=\|y\|^{-1}\left(t_{1} M_{1}+t_{2} M_{2}+\cdots+t_{K} M_{K}\right)-\|y\|^{-3} t^{T} y X_{0} y=0,
$$

where $k=1,2, \ldots, N-L$.

Let $X_{0}=\left[M_{1}, M_{2}, \ldots, M_{K}\right],(11)$ can be simplified as

$$
\frac{t \cdot y}{\|y\|} X_{0} y=X_{0} t \text {. }
$$

Substituting $y=X_{0}^{T} f$ into (12) it has

$$
\frac{t \cdot y}{\|y\|^{2}} f=\left(X_{0} X_{0}^{T}\right)^{-1} X_{0} t \text {. }
$$

The solution to (13) is the optimal group of filters and can be written as

$$
f=\left(X_{0} X_{0}^{T}\right)^{-1} X_{0} t
$$

Feed (14) into $y=X_{0}^{T} f$, the reconstructed signal $y$ can be obtained.

2.3. Processing Flow of Proposed Method. Due to the large mass and volume of the rotor and stator, the faulty vibration signal of the generator appears to be more stable so that the characteristic components are not so evident. For multipole generators, the components are even richer since the fundamental mechanical frequency (the rotating frequency of the rotor) is smaller (signal components should be multiple times of the fundamental frequency). In this case, the signal to noise ratio (SNR) is usually low and the fault diagnosis (especially when the fault is under a light degree) is always hard.

To solve the very problem, in this paper, we propose the method which combines EWES with MOMEDA for the characteristic component enhancement and the fault detection. The key flow is illustrated in Figure 1, and the detailed process is as follows:

(1) Calculate the wavelet time-energy spectrum of the original signal, using the Morlet wavelet as the basis function.

(2) Calculate the value of $T$. $T$ is the period of the deconvolution. For the generator studied in this paper, the fundamental frequency $f_{0}$ is $50 \mathrm{~Hz}$, while the sampling frequency $f_{s}$ is $5000 \mathrm{~Hz}$.

$$
T=\frac{f_{s}}{f_{0}}=\frac{5000 \mathrm{~Hz}}{50 \mathrm{~Hz}}=100 .
$$

(3) Enhance the impulse components in the wavelet time-energy spectrum by MOMEDA.

(4) Diagnose the fault based on the signal spectrum. According to $[25,26]$, the key point for the fault identification and diagnosis lies in the amplitude comparison in the 2nd harmonic and the 4th harmonic. As the occurrence and the increment of rotor interturn short circuit, the 2 nd harmonic will be decreased, while the 4 th harmonic will be increased.

\section{Application to Simulating Signal}

In order to validate the proposed algorithm, a group of simulating signal is employed for processing comparison. According to $[25,26]$, the faulty signal includes vibration harmonics at $f, 2 f, 3 f$, and $4 f(f$ is the fundamental frequency of the electricity and is $50 \mathrm{~Hz}$ in this paper).

$$
\left\{\begin{array}{l}
y(t)=g(t)+n(t), \\
g(t)+0.1 \sin (2 \pi * 50 t)+0.1 \sin (2 \pi * 100 t)+0.1 \sin (2 \pi * 150 t)+0.1 \sin (2 \pi * 200 t), \\
n_{0}(t)=\text { rand }(\text { length }(g(t))), \\
n_{1}(t)=n_{0}(t)-\operatorname{mean}\left(n_{0}(t)\right), \\
n(t)=10 * n_{1}(t),
\end{array}\right.
$$




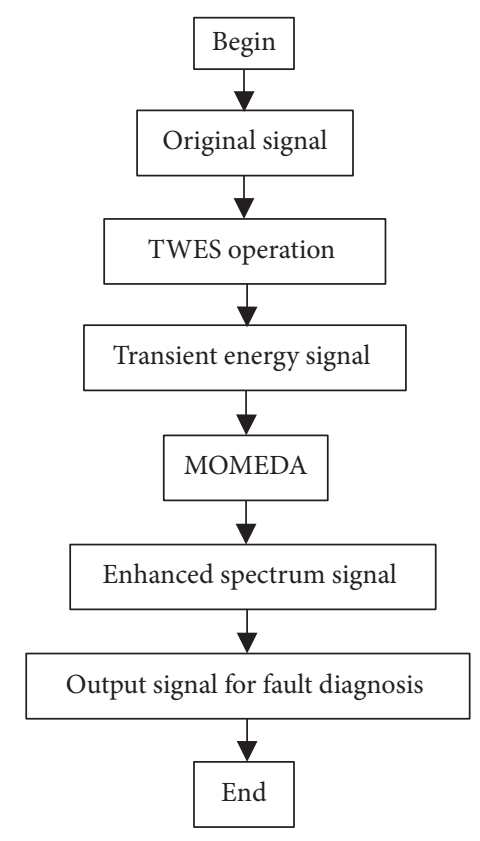

Figure 1: Flow chart of the algorithm.

where $g(t)$ is the characteristic signal, $\operatorname{rand}($ length $(g(t)))$ is the random function to generate the noise set which has the same data amount as $g(t)$, and $n(t)$ is the noise signal. The amplitudes of the characteristic signal and the noise signal are set by taking the reference of the actual vibration signal. The simulating signal without processing is illustrated in Figure 2(a).

As shown in Figure 2(a), the noise completely covers the characteristic signal. The simulating signal processed by the Time-wavelet energy spectrum (TWES) method, the MED method, the maximum correlative kurtosis deconvolution (MCKD) method [28], the EMD method, and the proposed method are illustrated in Figures 2(b)-2(f), respectively. During processing by MCKD and MED, the parameter $T$ is set to 100 (see equation (15)), and the filter length is set to 40 . These two parameters are set by taking the reference in [28]. For TWES, the window length is set to 30 , and the $n$-level is set to 512 [6]. For MOMEDA, the filter size $L=1000$ [19], the period $T$ is set to 100 (see equation (15)). The same parameters will be used behind in the test vibration signal processing.

As shown in Figures 2(b)-2(e), the characteristic harmonics, namely, the 1st $(50 \mathrm{~Hz}), 2 \mathrm{nd}(100 \mathrm{~Hz}), 3 \mathrm{rd}(150 \mathrm{~Hz})$, and the 4th $(200 \mathrm{~Hz})$ harmonics, are not prominent after processing by the TWES spectrum method, the MED method, the MCKD method, and the EMD method. On the contrary, the proposed method can effectively remove the noise signal and enhance the spectrum to highlight the characteristic harmonics. The proposed method displays a superior result to the other aforementioned methods.

\section{Practical Application to Experimental Generator}

4.1. Experimental Signal Acquisition. The experimental vibration signal is tested and sampled from the stator of a MJF-
30-6 prototype generator in State Key Laboratory of Alternate Electrical Power Systems with Renewable energy Sources, China, as shown in Figure 3(a). The primary parameters of the generator are listed in Table 1.

The generator is driven by a Z2-91 type DC motor, as illustrated in Figure 3(a), while the slot and winding distribution are indicated in Figure 3(b). During the experiment, the generator is taking about $500 \mathrm{~W}$ load. The exciting voltage is $95 \mathrm{~V}$, and the exciting current is $1 \mathrm{~A}$. The stator vibration signal is sampled by the velocity sensors (the sensitivity is $30 \mathrm{mv} / \mathrm{mm} / \mathrm{s}$ ), setting the sampling frequency as $5000 \mathrm{~Hz}$ which is 100 times of the fundamental frequency, as illustrated in Figure 3(c).

Three groups of data are sampled:

(1) Normal condition: The generator is running in normal condition, and the stator vibration signal is collected as the reference for further comparison.

(2) $1.5 \%$ rotor interturn short circuit fault: The exciting windings in the rotor are manually shorted by connecting the short circuit taps $\mathrm{C} 1 \mathrm{C} 2$ on the generator with a rheochord, see Figure 3(a).

(3) $2.5 \%$ rotor interturn short circuit fault: This is another faulty condition, with a severer faulty degree. The interturn short circuit degree is controlled by adjusting the value of the rheochord and calculated via

$$
F_{d}=\frac{I_{f}^{\prime}}{I_{f}} \times 25 \%,
$$

where $I_{f}^{\prime}$ is the short circuit current, while $I_{f}$ is the exciting current. For Group (2), the short circuit current is $0.06 \mathrm{~A}$ (1.5\%) while for Group (3) the short circuit current is $0.1 \mathrm{~A}$ (2.5\%).

4.2. Results and Discussion. The original vibration signal of normal condition, $1.5 \%$ rotor interturn short circuit, and $2.5 \%$ rotor interturn short circuit without any processing is illustrated in Figure 4 as a reference. In the meantime, the stator vibration signal is processed by TWES, MED, EMD, MOMEDA, MCKD, and the proposed method, respectively, see Figures 5-10. In theory, the maximum frequency of the spectrum should be equal to the half of the sampling frequency (the left side and the right side of the spectrum should be symmetric). Since the amplitudes of the higher order harmonics are comparatively much smaller than the characteristic components, in this paper, we set the max frequency to $400 \mathrm{~Hz}$ which is twice of the max characteristic component's frequency, to show the result with a proper resolution. Also, to show the time-domain waves under a proper resolution, we set the maximum time as $0.6 \mathrm{~s}$ which is thirty times of the period (the fundamental frequency is $50 \mathrm{~Hz}$ and the period is $0.02 \mathrm{~s}$ ).

Specifically, as indicated in Figures 4-10, the mean values of the processed signal in time-domain by different methods are different. This phenomenon reflects the varied noise- 

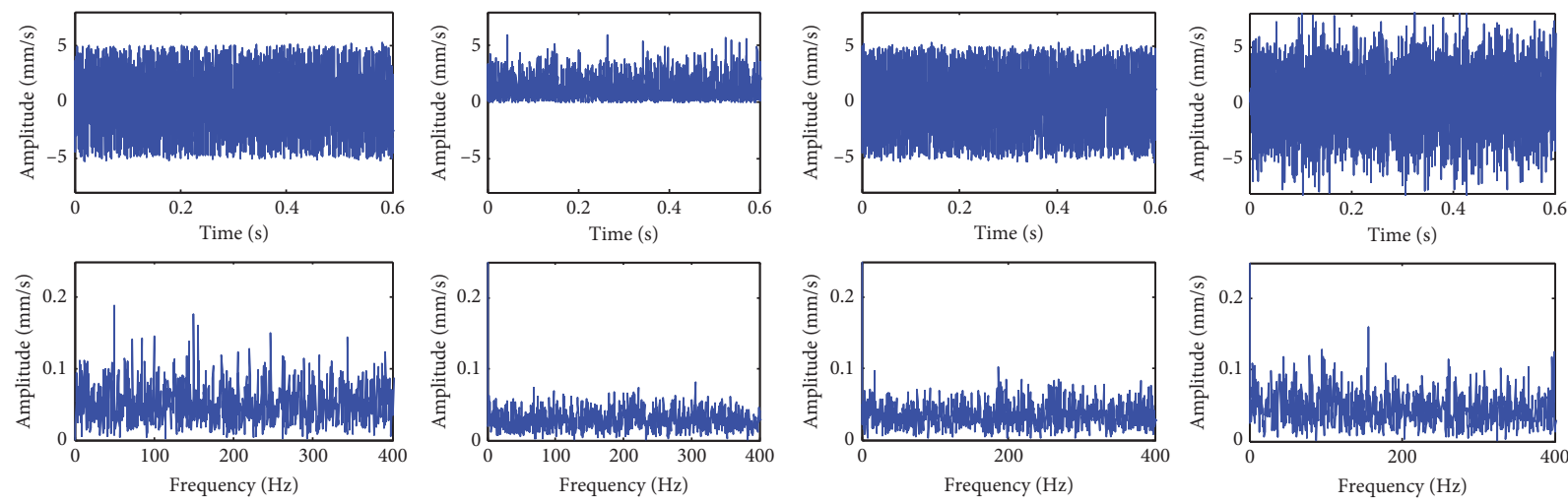

(a)

(b)

(c)

(d)
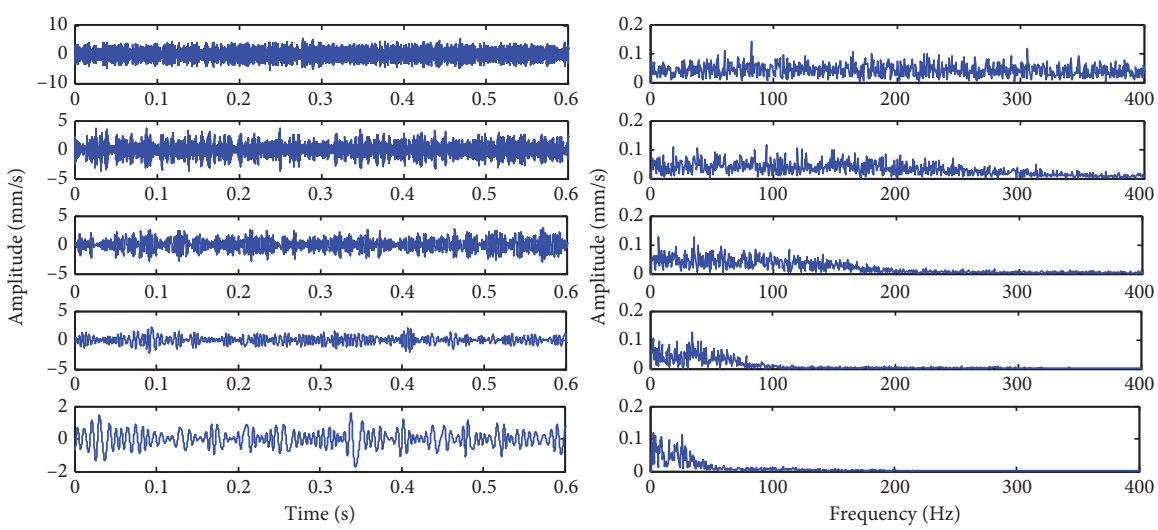

(e)
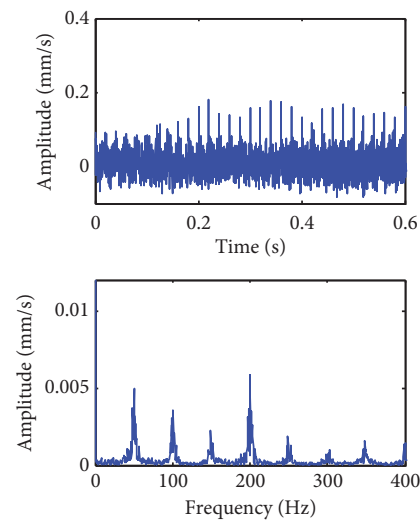

(f)

FIgURE 2: Simulating signal (a) without processing, (b) by the Time-wavelet energy spectrum method, (c) by the MED method, (d) by the MDCK method, (e) by the EMD method, and (f) by the proposed method.

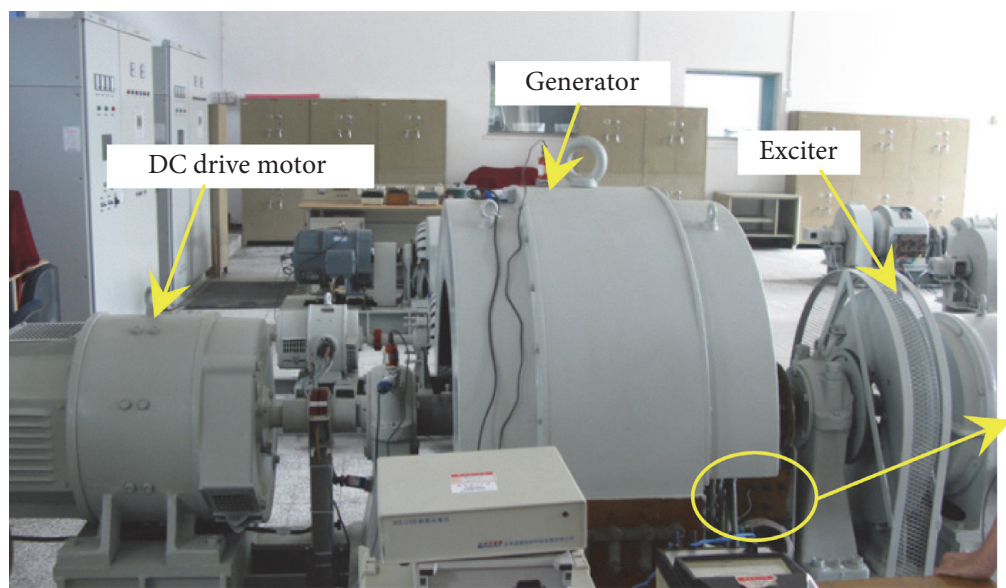

(a)

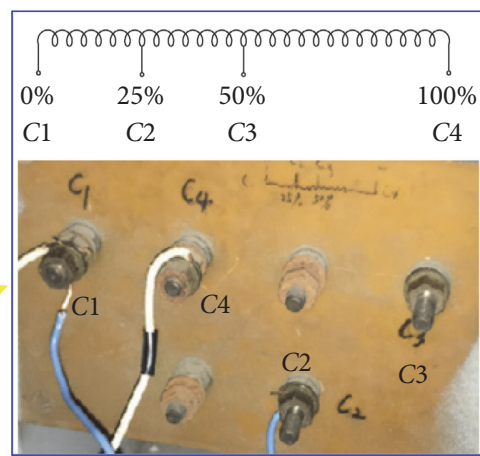

FIgURE 3: Continued. 


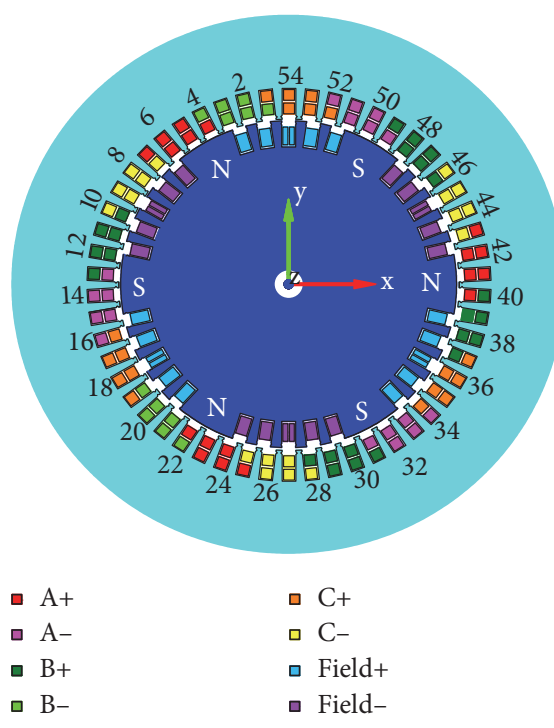

(b)

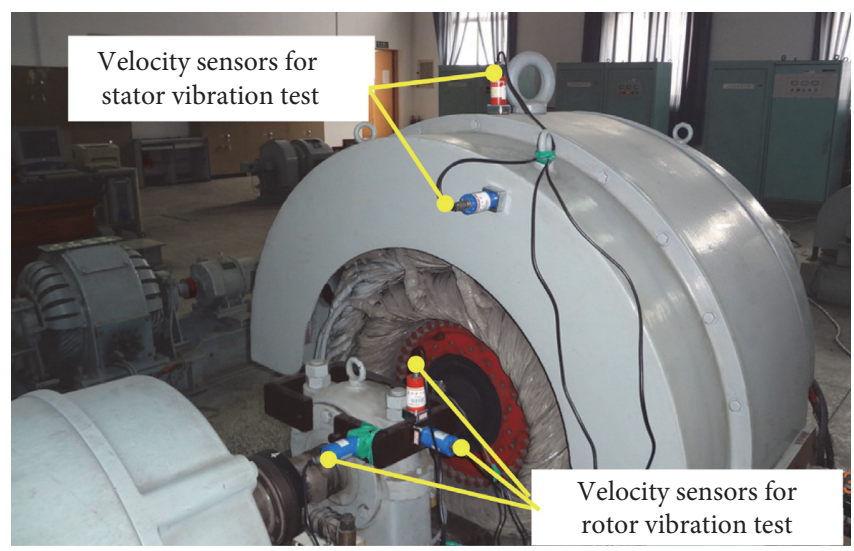

(c)

FIGURE 3: MJF-30-6 type nonsalient fault simulating generator: (a) picture of generator and rotor interturn short circuit taps, (b) slot and winding distribution, and (c) sensor set to test vibration signal.

TABLE 1: Primary parameters of MJF-30-6 prototype generator.

\begin{tabular}{lc}
\hline Parameter & Value \\
\hline Rated capacity & $30 \mathrm{kVA}$ \\
Stator slots & 54 \\
Rated rotating speed & $n_{\mathrm{r}}=1000 \mathrm{r} / \mathrm{min}$ \\
Radial air-gap & $0.85 \mathrm{~mm}$ \\
Synchronous reactance & $x_{\mathrm{s}}=2.0308 \Omega$ \\
Rated voltage & $400 \mathrm{~V}$ \\
Slot number per phase per pole & 3 \\
Pole-pairs & $p=3$ \\
Stator winding turns in series & 72 \\
Power factor & 0.8 \\
\hline
\end{tabular}

removing effect as well as the characteristic signal enhancement effect of different methods. On one hand, when the value of the noise (whose mean value is not zero) is much larger than the characteristic signal, i.e., in a very low RSN ratio case, the noise-removing degree will directly affect the mean value. On the other hand, the processing algorithm itself will also lead to varied mean values. For example, during the TWES processing, the energy spectrum is actually the absolute value, which means the mean value as well as the transient value will be positive, see Figures 2(b) and 5 .

As indicated in Figure 4, there is no significant difference in both the time-domain waves and the spectra between the normal and the faulty conditions. The characteristic components at $50 \mathrm{~Hz}(f), 100 \mathrm{~Hz}(2 f), 150 \mathrm{~Hz}(3 f)$, and $200 \mathrm{~Hz}$ (4f) are completely covered by the noises. In this case, it is pretty hard to identify the fault.

The processed results by TWES and MED are illustrated in Figures 5 and 6, respectively. It is shown in these two figures that there are differences between the normal and the faulty signals, and the amplitudes of the characteristic components are superior to others. This means TWES and
MED have the qualified function in characteristic component enhancement. Consequently, a coarse fault assessment can be made. However, the noise amplitude is still evident, and the processing result for the components at $150 \mathrm{~Hz}$ and $200 \mathrm{~Hz}$ is not satisfied.

The processed result by EMD is illustrated in Figure 7. It is shown that the four characteristic harmonics as well as their developing tendencies are not evident. However, the result still displays the effectiveness of EMD in compressing the noise and highlighting the key components, especially at $100 \mathrm{~Hz}$ and $200 \mathrm{~Hz}$. Although this method has obtained satisfied effect in the fault diagnosis for rolling bearings, it is not sufficient for the rotor interturn short circuit fault identification in multipole generators.

The processed results by MOMEDA, MCKD, and the proposed method are illustrated in Figures 8-10, respectively. Comparatively, the spectra by these three methods are pretty clean, and the amplitudes are concentrated in the multiple times of the fundamental frequency. This means the aforementioned three methods have the qualified noise filtering effect. Comparing with Figures 5-7, a more accurate fault assessment can be obtained. However, it is suggested in Figure 8 that the MOMEDA method is somewhat overfiltering, since the amplitudes of the characteristic harmonics are too small. Consequently, the amplitude differences between varied fault degrees are not evident enough. For instance, the amplitudes at $50 \mathrm{~Hz}$ for the three running conditions seem to be generally the same. On the contrary, the MCKD method and the proposed method show a clearer tendency in the characteristic harmonic variations. In another word, the proposed method as well as MCKD has the better processing effect for the experimental vibration signal.

For a better comparison, the amplitudes of each characteristic harmonic before and after rotor interturn short 

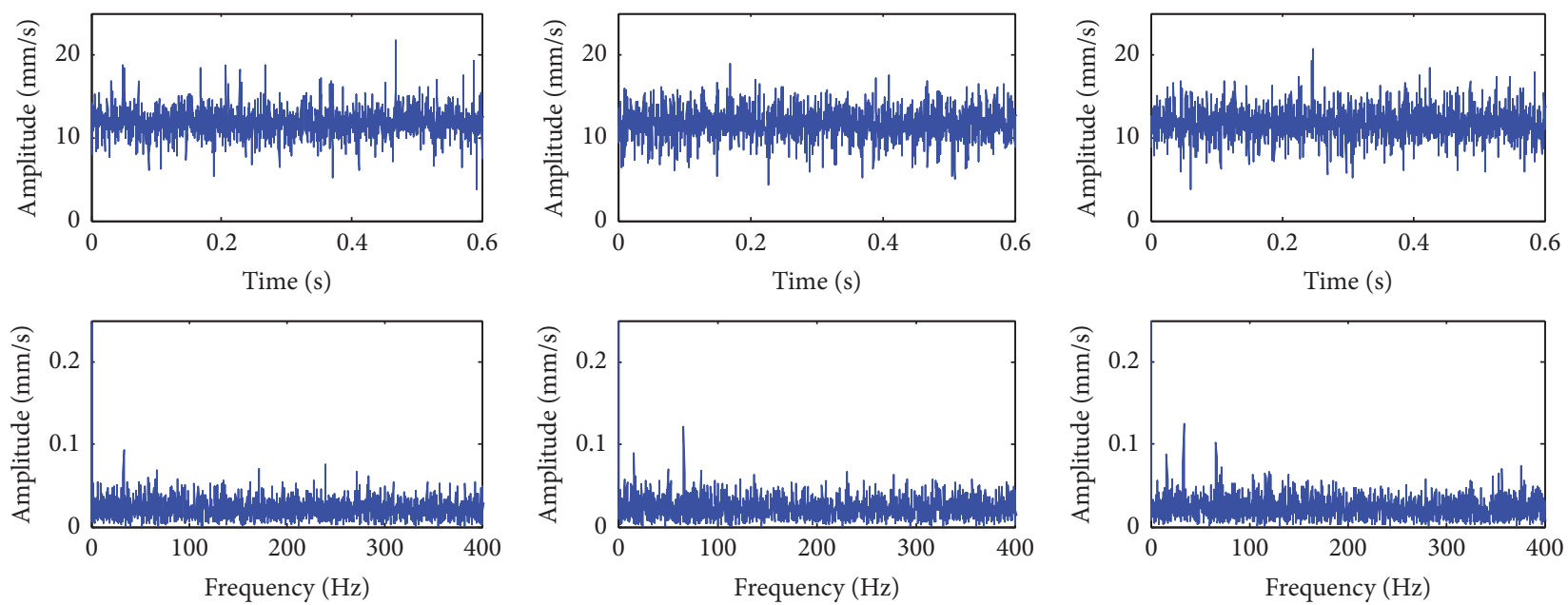

(a)

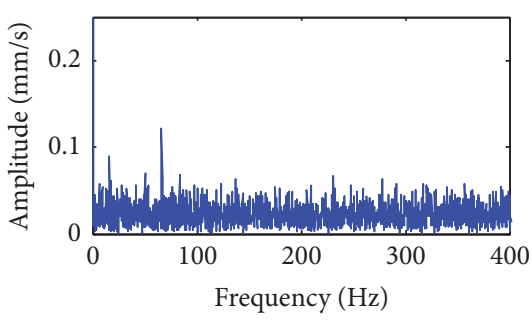

(b)

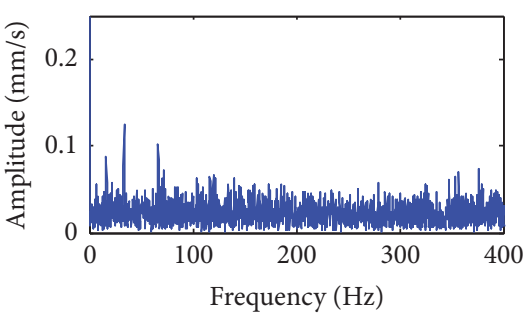

(c)

Figure 4: Original waves and spectra without any processing: (a) normal, (b) 1.5\% rotor short circuit, (c) $2.5 \%$ rotor short circuit.
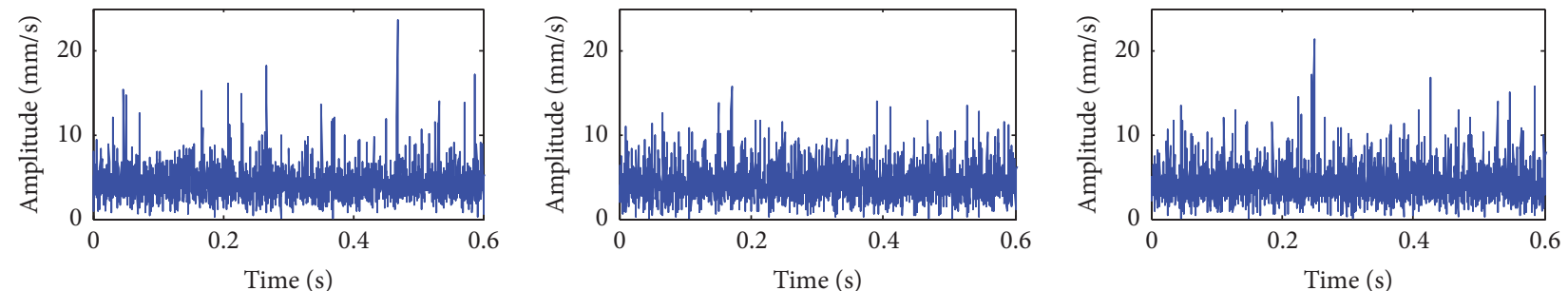

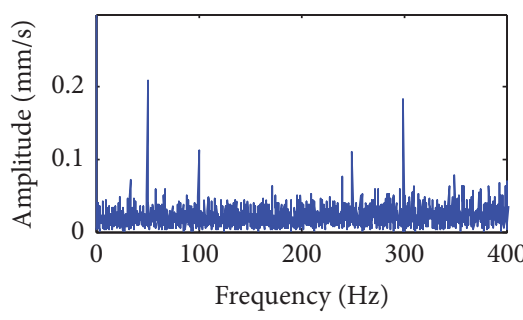

(a)

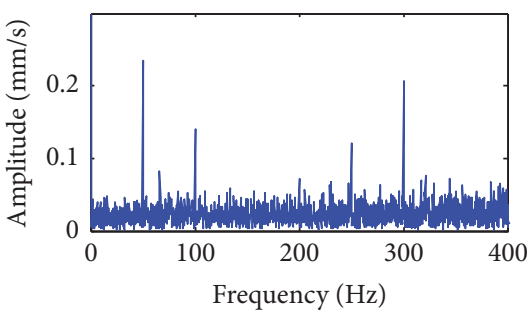

(b)

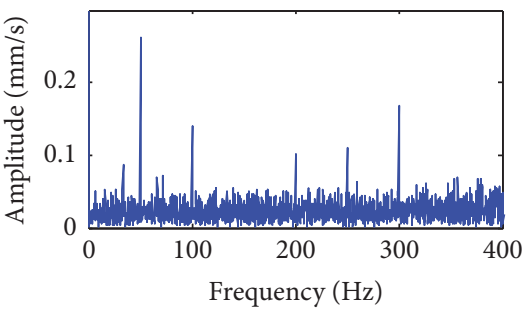

(c)

Figure 5: Waves and spectra processed by TWES: (a) normal, (b) $1.5 \%$ short circuit, (c) $2.5 \%$ short circuit.
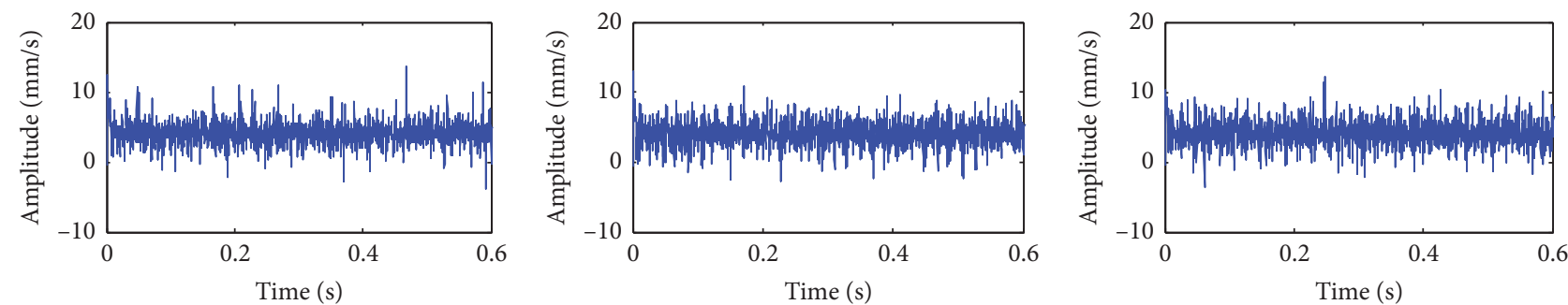

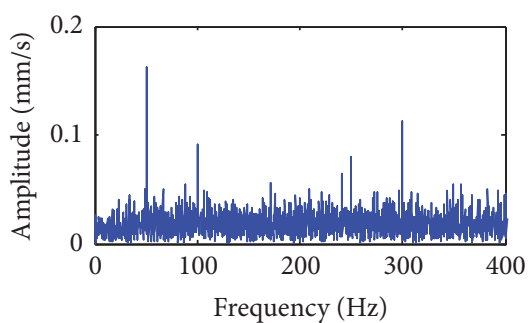

(a)

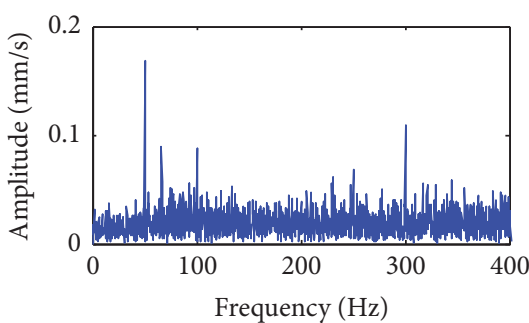

(b)

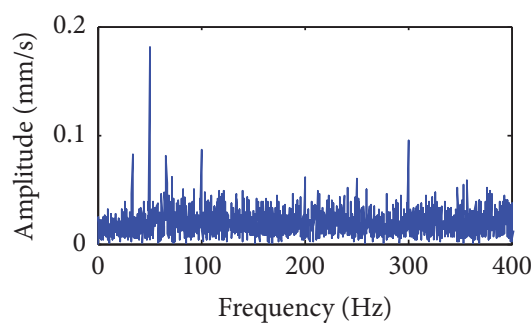

(c)

FIgURE 6: Waves and spectra processed by MED: (a) normal, (b) 1.5\% rotor short circuit, (c) 2.5\% rotor short circuit. 


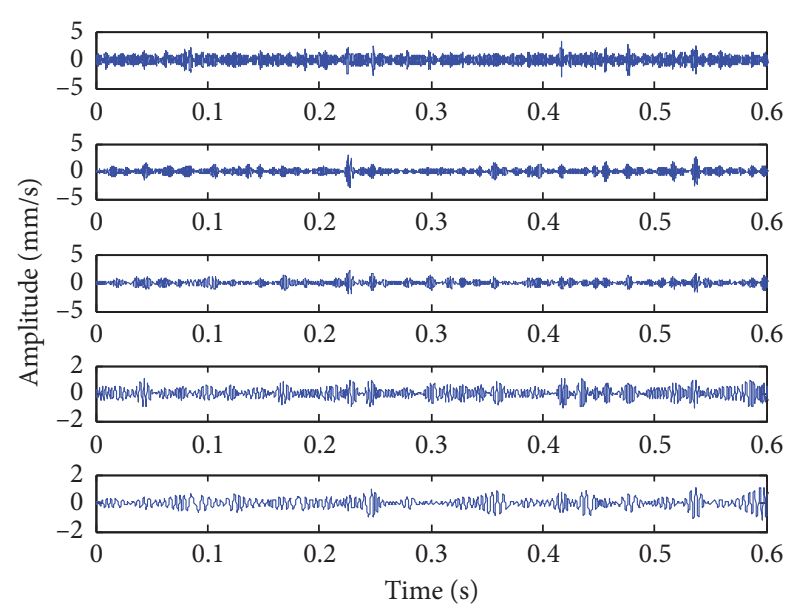

(a)
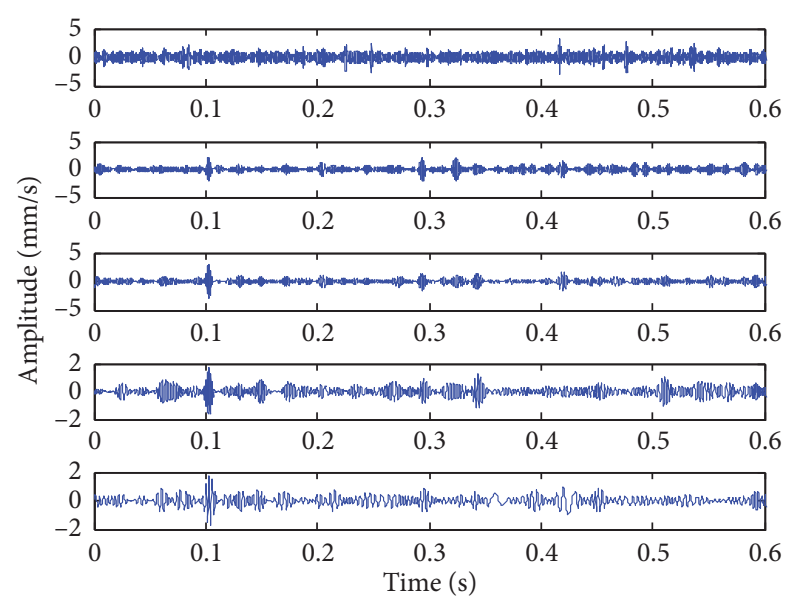

(b)
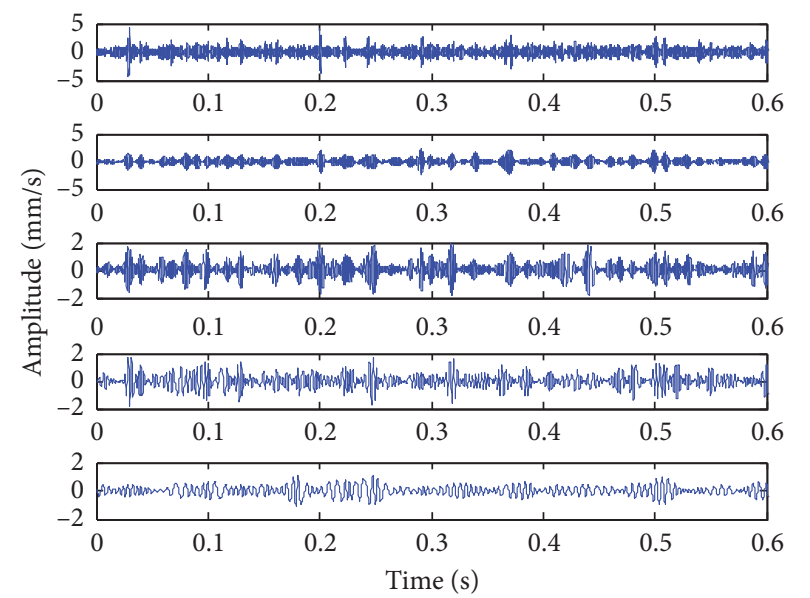

(c)

FIGURE 7: Waves and spectra processed by EMD: (a) normal, (b) 1.5\% rotor short circuit, (c) 2.5\% rotor short circuit.
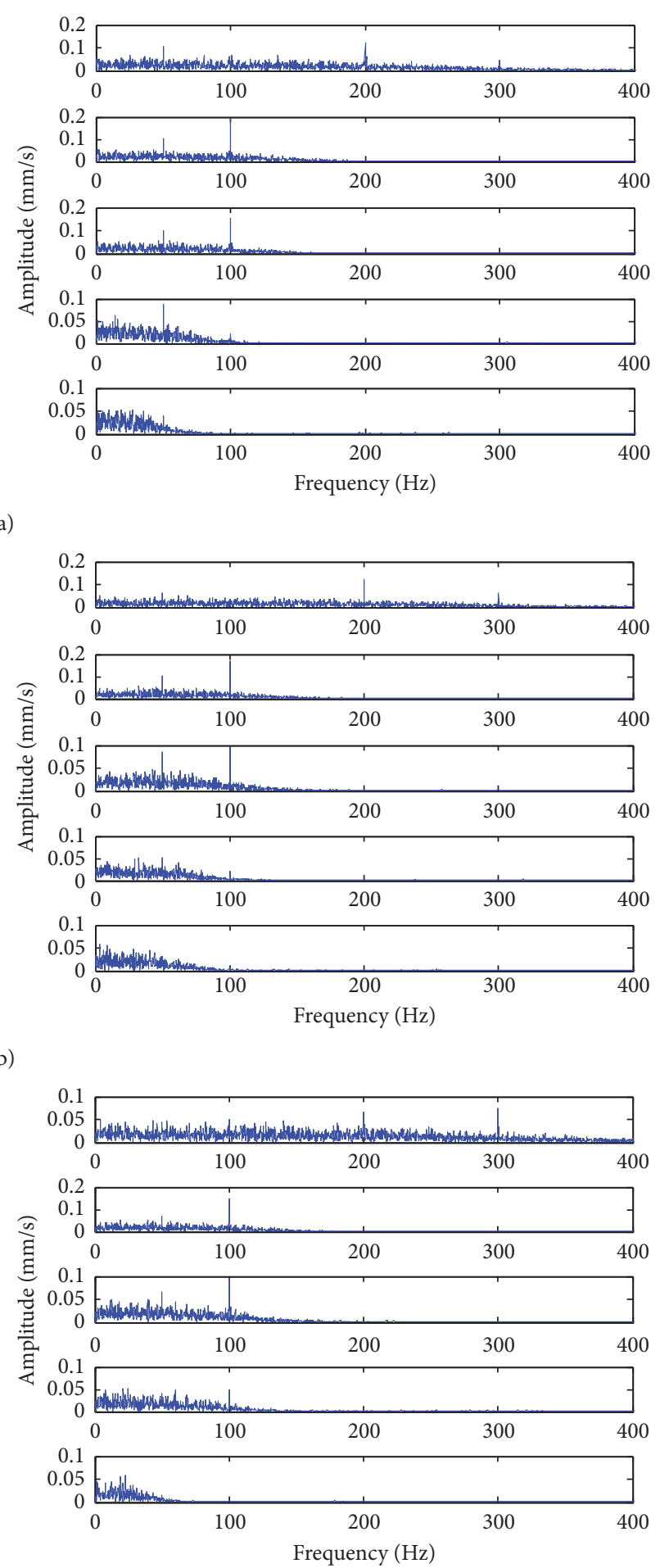

circuit by the proposed method and MCKD are listed in Table 2. As indicated in Table 2, by using the proposed method, the 2nd harmonic of the stator vibration shows a decreasing tendency, while the 1st, the $3 \mathrm{rd}$, and the 4th harmonics, especially the 4th harmonic, are showing an increasing tendency. This harmonic developing phenomenon accords well with $[25,26]$. However, the processing result by MCKD does not show such a developing tendency. The amplitude variation of the four characteristic components cannot match well with the theoretical developing trend and therefore will cause confusion to the technical people. Consequently, the rotor interturn short 

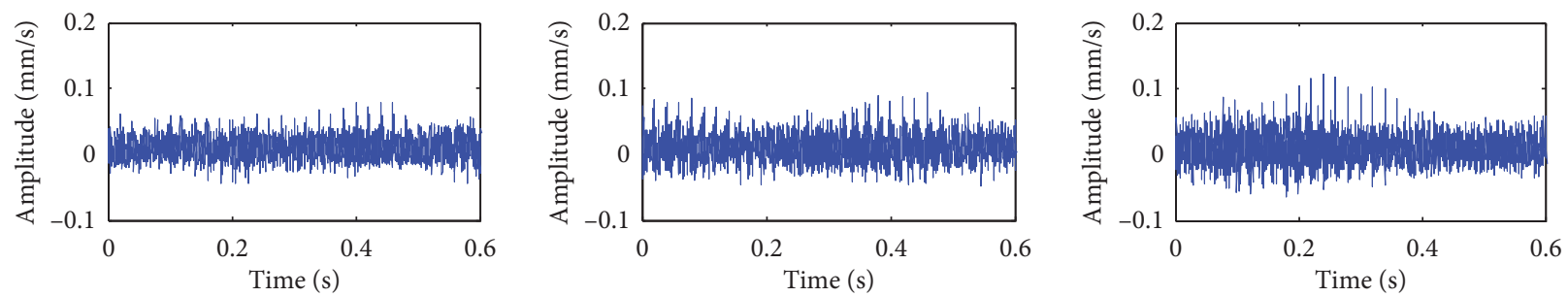

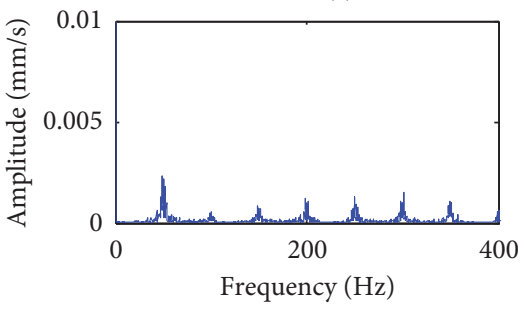

(a)

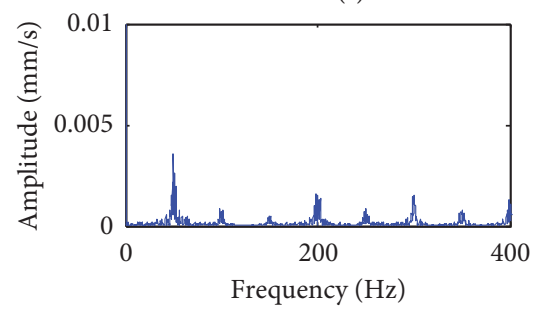

(b)

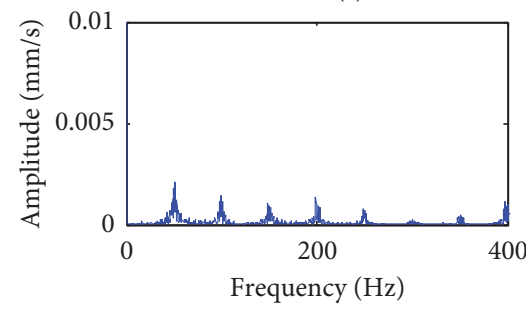

(c)

FIgURE 8: Waves and spectra processed by MOMEDA: (a) normal, (b) $1.5 \%$ rotor short circuit, (c) $2.5 \%$ rotor short circuit.
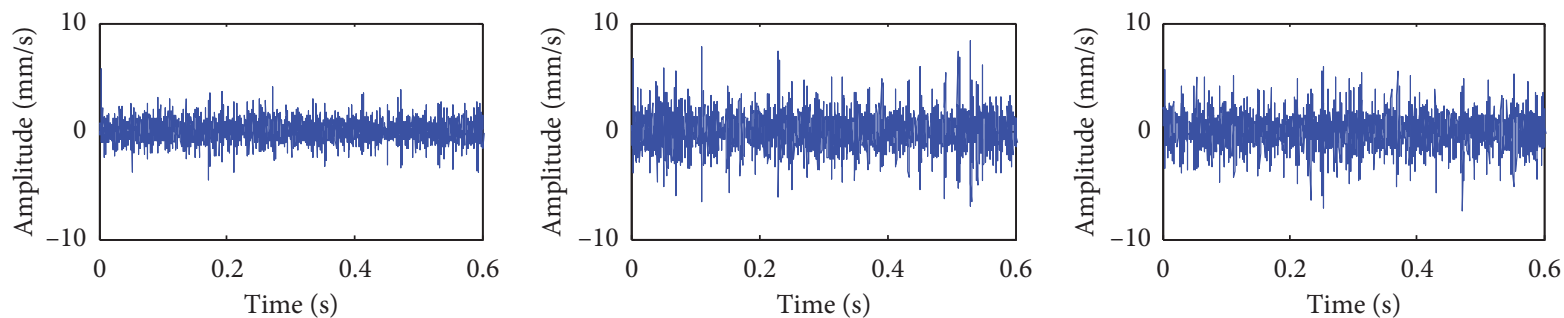

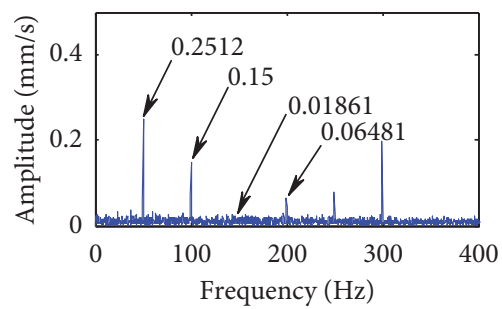

(a)

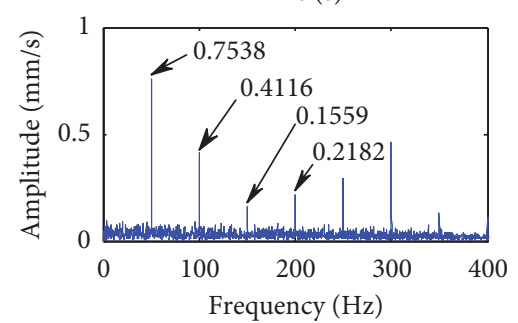

(b)

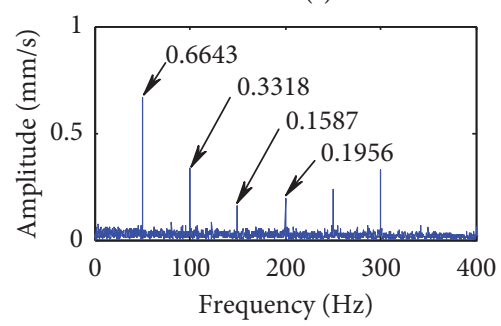

(c)

FIGURE 9: Waves and spectra processed by MCKD: (a) normal, (b) 1.5\% rotor short circuit, (c) 2.5\% rotor short circuit.
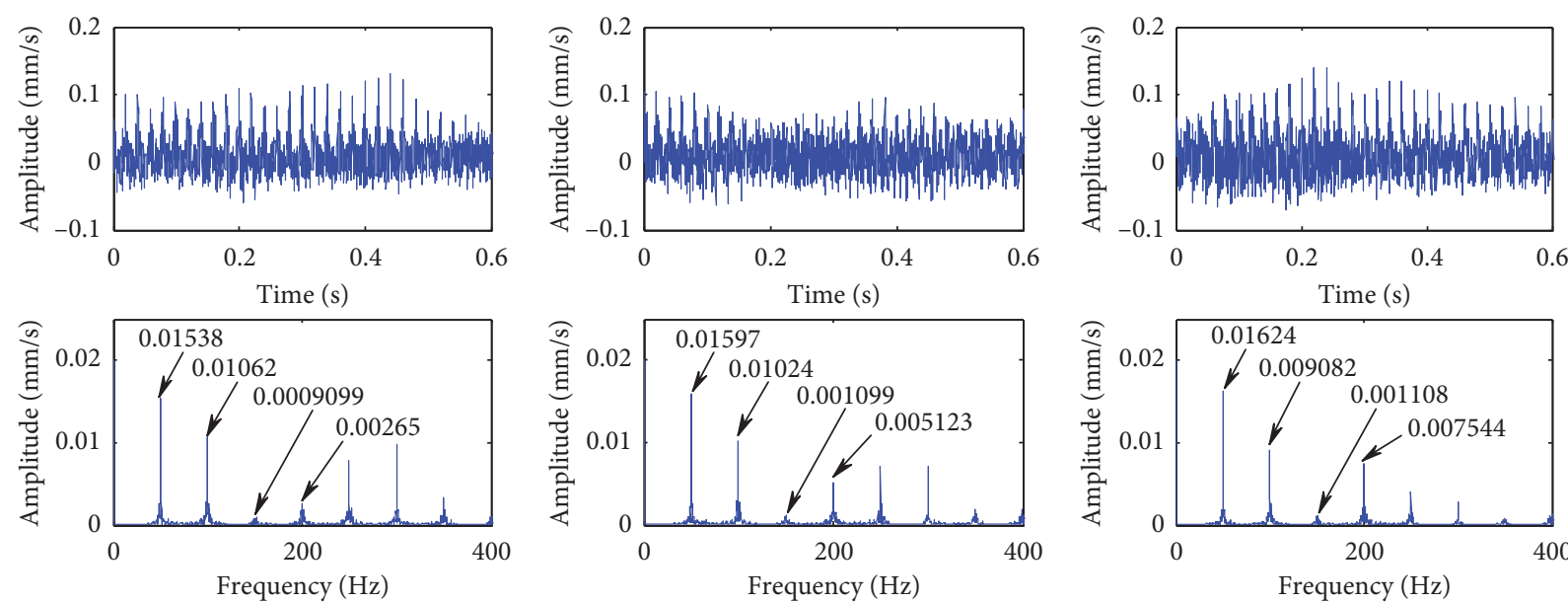

(a)

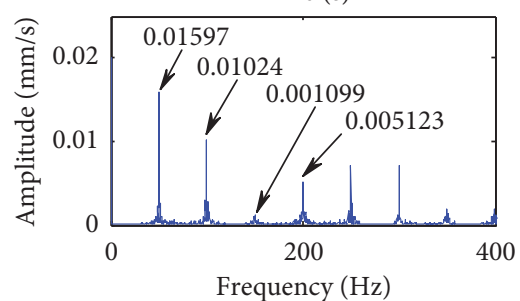

(b)

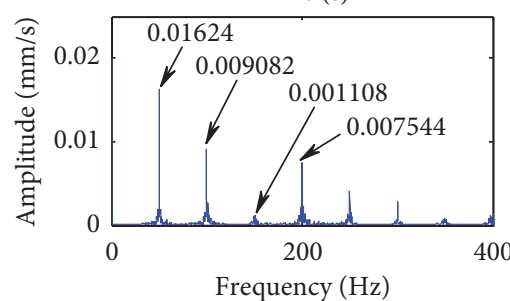

(c)

FigURE 10: Waves and spectra obtained by the proposed method: (a) normal, (b) 1.5\% rotor short circuit, (c) $2.5 \%$ rotor short circuit. 
TABLE 2: Stator radial vibration amplitude.

\begin{tabular}{lcccccc}
\hline & \multicolumn{3}{c}{ Processing method } \\
Stator vibration harmonic & \multicolumn{3}{c}{ Proposed method in this paper } & \multicolumn{2}{c}{ MCKD } \\
& Normal & $1.5 \%$ short circuit & $2.5 \%$ short circuit & Normal & $1.5 \%$ short circuit & $2.5 \%$ short circuit \\
\hline$F$ & 0.01538 & 0.01597 & 0.01624 & 0.2512 & 0.7538 & 0.6643 \\
$2 f$ & 0.01062 & 0.01024 & 0.009082 & 0.15 & 0.4116 & 0.3318 \\
$3 f$ & 0.0009099 & 0.001099 & 0.001108 & 0.01861 & 0.1559 & 0.1587 \\
$4 f$ & 0.00265 & 0.005123 & 0.007544 & 0.06481 & 0.2182 & 0.1956 \\
\hline
\end{tabular}

circuit cannot be accurately identified by this method. Thus, it can be concluded that the proposed method is superior to MCKD in diagnosing the rotor interturn short circuit fault based on the vibration signal in multipole generators.

In addition to the processing effect, the consuming time is also significant. For the same data processing (with the signal data length of 24992), the detailed consuming time for TWES, MED, EMD, MOMEDA, MCKD, and the proposed method is $2.8346 \mathrm{~s}, 0.2015 \mathrm{~s}, 4.2719 \mathrm{~s}, 2.6584 \mathrm{~s}, 0.4155 \mathrm{~s}$, and $2.8614 \mathrm{~s}$, respectively. The MED method consumes the least time, while the EMD algorithm consumes the most. The proposed method consumes much more time than MED and MCKD but $35 \%$ less time than EMD, while generally similar time as TWES and MOMEDA.

On the other hand, it should be noted that there is also shortcoming in the proposed method. The vibration amplitudes after processing are much smaller than the actual ones, though the spectra structure has been enhanced. This amplitude compressing effect is primarily caused by MOMEDA (see Figure 8), since the proposed method combines TWES and MOMEDA. A possible quick way to modify the amplitudes is to multiply a coefficient to all of the amplitude values for each harmonic. However, to find what is the exact value of the coefficient needs further investigation. We will carry out the further work in this topic in the short future.

\section{Conclusion}

In this paper, we propose a new vibration signal processing method for the rotor interturn short circuit fault identification in multipole generators. This proposed method combines the Time-Wavelet Energy Spectrum (TWES) with the multipoint optimal minimum entropy deconvolution adjusted (MOMEDA) algorithm, to filter the noise meanwhile enhancing the characteristic components.

The primary contribution/novelty of this paper lies in two aspects: (1) an effective processing method for the pretty low SNR stator vibration signal in multipole generator has been proposed. This method, which well highlights the characteristic harmonics and removes the noise, shows the actual fault developing tendency and is superior to some other general methods such as EMD, MED, MCKD, etc., and (2) differently from most other researches, in this paper, the stator vibration signal for the particular rotor interturn short circuit fault of multipole generator is employed as the study object, while most previous studies concentrated in the vibration signals of rolling bearings/rotors. The research work in this paper is beneficial for the fast fault identification for the rotor interturn short circuit fault, since it is more convenient to collect the stator vibration signal than the rotor vibration signal (for example, in wind turbine generators, the bearings are covered inside the stator housing and the bearing/rotor vibration signal are not so easy to collect).

Based on the study in this paper, it is found that the original vibration signal without any processing is far away from the accurate fault diagnosis since the key characteristic components are completely covered by the noise. The TWES algorithm has a good signal enhancement property for the characteristic components, while MOMEDA method has the qualified noise filtering effect. However, neither of these two methods is satisfied enough for the exact fault detection. Comparatively, the proposed method in this paper which combines these two methods together can not only filter the noise but also enhance the characteristic components properly. This method is of high potential to be carried out for the practical application.

\section{Data Availability}

The data used to support the findings of this study are available from the corresponding author upon request.

\section{Conflicts of Interest}

All of the authors declare that there are no interest conflicts regarding the publication of this paper.

\section{Acknowledgments}

This work was in part supported by National Natural Science Foundation of China (51777074), the Hebei Provincial Natural Science Foundation (E2019502047), the Chinese Fundamental Research Funds for the Central Universities (2017MS152 and 2018YQ03), and The 3rd Top Youth Talent Support Program of Hebei Province ([2018]-27).

\section{References}

[1] H. Ma, T. Chen, Y. Zhang, P. Ju, and Z. Chen, "Research on the fault diagnosis method for slip ring device in doubly-fed induction generators based on vibration," IET Renewable Power Generation, vol. 11, no. 2, pp. 289-295, 2017.

[2] M. Cuevas, R. Romary, J.-P. Lecointe, and T. Jacq, "Noninvasive detection of rotor short-circuit fault in synchronous machines by analysis of stray magnetic field and frame vibrations," IEEE Transactions on Magnetics, vol. 52, no. 7, pp. 1-4, 2016.

[3] Z. Huo, Y. Zhang, P. Francq, L. Shu, and J. Huang, "Incipient fault diagnosis of roller bearing using optimized wavelet transform based multi-speed vibration signatures," IEEE Access, vol. 5, pp. 19442-19456, 2017. 
[4] J. Seshadrinath, B. Singh, and B. K. Panigrahi, "Incipient turn fault detection and condition monitoring of induction machine using analytical wavelet transform," IEEE Transactions on Industry Applications, vol. 50, no. 3, pp. 2235-2242, 2014.

[5] J. Zhang, H. Sun, Z. Sun, W. Dong, and Y. Dong, "Fault diagnosis of wind turbine power converter considering wavelet transform, feature analysis, judgment and BP neural network," IEEE Access, vol. 7, pp. 179799-179809, 2019.

[6] Y. Xin, S. Li, Z. Zhang, Z. An, and J. Wang, "Adaptive reinforced empirical morlet wavelet transform and its application in fault diagnosis of rotating machinery," IEEE Access, vol. 7, pp. 65150-65162, 2019.

[7] J. A. Antonino-Daviu, M. Riera-Guasp, J. Pons-Llinares, J. Roger-Folch, R. B. Perez, and C. Charlton-Perez, "Toward condition monitoring of damper windings in synchronous motors via EMD analysis," IEEE Transactions on Energy Conversion, vol. 27, no. 2, pp. 432-439, 2012.

[8] Y. Li, M. Xu, X. Liang, and W. Huang, "Application of bandwidth EMD and adaptive multiscale morphology analysis for incipient fault diagnosis of rolling bearings," IEEE Transactions on Industrial Electronics, vol. 64, no. 8, pp. 6506-6517, 2017.

[9] Z. Jiang, Y. Wu, J. Li, Y. Liu, J. Wang, and X. Xu, "Application of EMD and 1.5-dimensional spectrum in fault feature extraction of rolling bearing," The Journal of Engineering, vol. 2019, no. 23, pp. 8843-8847, 2019.

[10] L. Dong, H. Zeng, Z. Xiao et al., "Fault diagnosis of rotor using EMD thresholding-based denoising combined with probabilistic neural network," Journal of Vibroengineering, vol. 19, no. 8, pp. 5920-5931, 2017.

[11] D. Liu, Z. Xiao, X. Hu, C. Zhang, and O. P. Malik, "Feature extraction of rotor fault based on EEMD and curve code," Measurement, vol. 135, pp. 712-724, 2019.

[12] W. Wang, W. Peng, M. Tian, and W. Tao, "Partial discharge of white noise suppression method based on EEMD and higher order statistics," The Journal of Engineering, vol. 2017, no. 13, pp. 2043-2047, 2017.

[13] A. Liao, C. Shen, and P. Li, "Potential contrast improvement in ultrasound pulse inversion imaging using EMD and EEMD," IEEE Transactions on Ultrasonics, Ferroelectrics, and Frequency Control, vol. 57, no. 2, pp. 317-326, 2010.

[14] X. Liu, X. Zhang, Z. Luan, and X. Xu, "Rolling bearing fault diagnosis based on EEMD sample entropy and PNN," The Journal of Engineering, vol. 2019, no. 23, pp. 8696-8700, 2019.

[15] J. Li, M. Li, and J. Zhang, "Rolling bearing fault diagnosis based on time-delayed feedback monostable stochastic resonance and adaptive minimum entropy deconvolution," Journal of Sound and Vibration, vol. 401, pp. 139-151, 2017.

[16] S. Abrar and A. K. Nandi, "Adaptive minimum entropy equalization algorithm," IEEE Communications Letters, vol. 14, no. 10, pp. 966-968, 2010.

[17] L. Zhang and N. Hu, "Fault diagnosis of sun gear based on continuous vibration separation and minimum entropy deconvolution," Measurement, vol. 141, pp. 332-344, 2019.

[18] Y. Cheng, N. Zhou, W. Zhang, and Z. Wang, "Application of an improved minimum entropy deconvolution method for railway rolling element bearing fault diagnosis," Journal of Sound and Vibration, vol. 425, pp. 53-69, 2018.

[19] G. L. Mcdonald and Q. Zhao, "Multipoint optimal minimum entropy deconvolution and convolution fix: application to vibration fault detection," Mechanical Systems and Signal Processing, vol. 82, pp. 461-477, 2017.

[20] Y. Cheng, B. Chen, and W. Zhang, "Adaptive multipoint optimal minimum entropy deconvolution adjusted and application to fault diagnosis of rolling element bearings," IEEE Sensors Journal, vol. 19, no. 24, pp. 12153-12164, 2019.

[21] C.J. Zhou, J. Ma, J. Wu, and Z. Feng, "A parameter adaptive MOMEDA method based on grasshopper optimization algorithm to extract fault features," Mathematical Problems in Engineering, vol. 2019, Article ID 7182539, 22 pages, 2019.

[22] Z. Wang, W. Du, J. Wang et al., "Research and application of improved adaptive MOMEDA fault diagnosis method," Measurement, vol. 140, pp. 63-75, 2019.

[23] Y. Xu, Z. Cai, X. Cai, and K. Ding, “An enhanced multipoint optimal minimum entropy deconvolution approach for bearing fault detection of spur gearbox," Journal of Mechanical Science and Technology, vol. 33, no. 6, pp. 2573-2586, 2019.

[24] H. Ma and Z. Feng, "Planet bearing fault diagnosis using multipoint optimal minimum entropy deconvolution adjusted," Journal of Sound and Vibration, vol. 449, pp. 235-273, 2019.

[25] Y.-L. He, K. Meng-Qiang, G.-J. Tang, H.-C. Jiang, and X.-H. Yuan, "Analysis and simulation on the effect of rotor interturn short circuit on magnetic flux density of turbogenerator," Journal Off Electrical Engineering, vol. 67, no. 36, pp. 323-333, 2016.

[26] G.-J. Tang, Y.-L. He, S.-T. Wan, and L. Xiang, "Investigation on stator vibration characteristics under air-gap eccentricity and rotor short circuit composite faults," Journal of the Brazilian Society of Mechanical Sciences and Engineering, vol. 36, no. 3, pp. 511-522, 2014.

[27] Y. Pan, L. Zhang, X. Wu, K. Zhang, and M. J. Skibniewski, "Structural health monitoring and assessment using wavelet packet energy spectrum," Safety Science, vol. 120, pp. 652-665, 2019.

[28] G. Tang, X. Wang, and Y. He, "Diagnosis of compound faults of rolling bearings through adaptive maximum correlated kurtosis deconvolution," Journal of Mechanical Science and Technology, vol. 30, no. 1, pp. 43-54, 2016. 